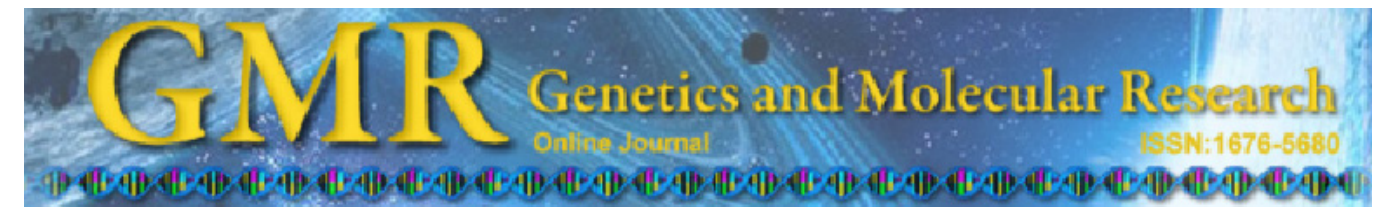

Methodology

\title{
Complete sequence analysis of mitochondrial DNA of aplastic anemia patients
}

X. Cui ${ }^{1}$, F. Liu ${ }^{2}$, J.Q. Wang ${ }^{3}$, W.J. Zhang ${ }^{4}$, J.Y. Wang ${ }^{1}$, K. Liu ${ }^{1}$, S.Y. Cui ${ }^{1}$, J. Zhang ${ }^{1}$ and R.R. $\mathrm{Xu}^{1}$

${ }^{1}$ Department of Hematology, Affiliated Hospital, Shandong University of Traditional Chinese Medicine, Jinan, China ${ }^{2}$ Department of Hematology, Yantaishan Hospital, Yantai, China ${ }^{3}$ Department of Health Care, Second Affiliated Hospital, Shandong University of Traditional Chinese Medicine, Jinan, China ${ }^{4}$ Department of Scientific Research, Affiliated Hospital,

Shandong University of Traditional Chinese Medicine, Jinan, China

Corresponding author: X. Cui

E-mail: cdz45@163.com

Genet. Mol. Res. 11 (3): 2130-2137 (2012)

Received May 17, 2012

Accepted June 22, 2012

Published August 6, 2012

DOI http://dx.doi.org/10.4238/2012.August.6.16

ABSTRACT. This study was primarily undertaken to test the hypothesis that mitochondrial DNA (mtDNA) mutations may be associated with aplastic anemia (AA). We analyzed mtDNA sequences from 15 patients with AA. The samples were obtained from bone marrow, and patients' oral epithelial cells were collected for normal tissue comparison. Total DNA was amplified by PCR after extraction, and these segments were then sent for sequencing. The results were compared with those of oral epithelial tissues as well as mtDNA sequences in the revised Cambridge Reference Sequence (rCRS) database. We detected 61 heteroplasmic mutations in 11 genes, including those encoding NADH dehydrogenase $(N D)$ 1-2 and 4-6, tRNA glutamic acid (TRNE), ribosomal RNA (RNR) 1 and 2, cytochrome c oxidase (COX1), cytochrome b (CYTB), and tRNA glycine $(T R N G)$; mutation rates were particularly high in ND2 
(34.4\%) and ND4 (21.3\%) in the patients' mtDNA genomes. The products of these genes are involved in oxidation in the respiratory chain, and a large number of homoplasmic mutations were found. Interestingly, these 162 polymorphisms were mostly in the D-loop DNA structure (54.3\%), in which numerous mutations associated with leukemia and myelodysplastic syndromes are found. We conclude that functional impairment of the mitochondrial respiratory chain induced by mutation may be an important reason for hematopoietic failure in AA patients.

Key words: Aplastic anemia; Mitochondrial DNA; Maternal inheritance; Mutation

\section{INTRODUCTION}

Aplastic anemia (AA) is a bone marrow failure syndrome characterized by peripheral pancytopenia and marrow hypoplasia. The damage to bone marrow may be triggered by environmental exposure, such as exposure to chemicals and drugs, or viral infections, and possibly, endogenous antigens generated by genetically altered bone marrow cells.

While most of a cell's genetic material is contained within the nucleus, mitochondria have their own circular DNA. They also have their own machinery for protein synthesis and reproduce by the process of fission, as in bacteria. Human mitochondrial DNA (mtDNA) is a $16-\mathrm{kb}$ circle that contains genes encoding 13 electron transport chain proteins, 22 tRNAs, and 2 rRNAs (Shadel and Clayton, 1997; DiMauro and Schon, 2003). Mitochondria are considered to be the "power houses of the cell" because they produce adenosine triphosphate (ATP) by systematically extracting energy from nutrient molecules (substrates) (Chinnery and Schon, 2003). mtDNA is replicated with a high mutation rate because it has no protective histones and also lacks an effective DNA repair system. Moreover, mtDNA is located near the inner mitochondrial membrane, where it is far more exposed to oxygen-free radicals generated by the respiratory chain than is nuclear DNA (Richter et al., 1988; Penta et al., 2001). Although the limited repair capacity hypothesis has been validated experimentally in some experimental systems, recent data have shown that base excision repair mechanisms do occur in mammalian mtDNA (Bohr et al., 2002; Chen et al., 2002).

Several laboratories have reported unexpectedly large numbers of somatic mutations in leukemia and myelodysplastic syndromes (Grist et al., 2004; Linnartz et al., 2004; Wulfert et al., 2008). Acquired deletions of mtDNA in the hematopoietic compartment have also been found to occur in association with severe pancytopenia and reticulocytopenia (Hatfill et al., 1993). We hypothesized that AA may be associated with mtDNA aberrations. In the present study, we analyzed the entire mtDNA nucleotide sequences from 15 patients with AA and found that there were high mutational rates in the genes encoding NADH dehydrogenase $(N D)$ 1-2 and 4-6, cytochrome B (CYTB) and related genes [tRNA glutamic acid (TRNE), ribosomal RNA (RNR) 1 and 2, cytochrome c oxidase (COXI), tRNA glycine (TRNG)], which are closely involved in oxidation in the respiratory chain. We were led to conclude that functional impairment of the mitochondrial respiration chain induced by gene mutation may be an important reason behind hematopoietic failure in AA. 


\section{PATIENTS AND METHODS}

\section{Patients}

Between September 2010 and February 2011, 15 patients, 7 males and 8 females (median age of 32.2 years; range 11-64), were included in this study. Eligible patients had histologically confirmed diagnosis of AA (Table 1 and Figure 1). These patients had no family history of hematologic disease, and most of them presented fatigue and petechiae. Bone marrow cellularity was low or extremely low in all patients. The karyotype was normal in all patients except patient 2, who was 45,XX,-7. This study was approved by the Institutional Review Board of the Affiliated Hospital of Shandong University of Traditional Chinese Medicine, and written informed consent was obtained from all participants in accordance with the Declaration of Helsinki.

\section{Genomic DNA extraction}

Bone marrow cells were collected from patients, and oral epithelial cells were collected for normal tissue comparison. Total DNA from bone marrow and oral epithelium was extracted using an EasyPure Genomic DNA Extraction kit (Beijing Tiangen Biotech Co., China). Extracted DNA was resuspended in Tris-EDTA buffer (10 mM Tris-HCl, $1 \mathrm{mM}$ EDTA, $\mathrm{pH} 7.5$ ) and stored at $4^{\circ} \mathrm{C}$ prior to use.

\section{mtDNA sequencing and data analysis}

For the direct sequencing of the entire mtDNA genome, we used 8 primer pairs based on a modification of a published protocol to obtain 8 partially overlapping segments. The amplified mtDNA PCR products were directly sequenced using the BigDye Terminator v3.1 ready reaction kit (Applied Biosystems) and the ABI Prism 3100 Genetic Analyzer (Applied Biosystems). Sequencing primers used for each mtDNA product are shown in Table 2, and the electropherogram of 8 fragments is shown in Figure 1.

Experimentally obtained mtDNA sequences were compared with the revised Cambridge Reference Sequence (rCRS) (http://www.mitomap.org/mitomap/mitoseq.html; Andrews et al., 1999) by using the Blast2 program (http://www.ncbi.nlm.nih.gov/blast/Blast.cgi?PAGE_ TYPE=BlastSearch\&PROG_DEF=blastn\&BLAST_PROG_DEF=megaBlast\&SHOW DEFAULTS $=$ on\&BLAST_SPEC $=$ blast2seq\&LINK_LOC $=$ align2seq) and the database search tool MitoAnalyzer (http://www.cstl.nist.gov/biotech/strbase/mitoanalyzer.html, 2001; Lee and Levin, 2002) to determine which polymorphisms and mutations differed from the rCRS and whether the differences caused amino acid changes in the resultant polypeptides. Nucleotide changes that were present in both the bone marrow and oral epithelial cells in the same patient were counted as polymorphisms or homoplasmic mutations, and those that had not already been included in the databases (MITOMAP, mtDB, or GenBank) were considered new polymorphisms. Changes that were only present in bone marrow were counted as mutations or heteroplasmic mutations.

\section{RESULTS}

\section{Heteroplasmic mutations of mtDNA}

We detected mutations in all 15 specimens, and overall, we detected 61 mutations in 


\begin{tabular}{|c|c|c|c|c|c|c|c|c|c|c|}
\hline Patient & $\begin{array}{l}\text { Gender/age } \\
\text { (years) }\end{array}$ & $\begin{array}{l}\text { Bone marrow } \\
\text { cellularity }\end{array}$ & $\begin{array}{l}\text { Hematopoietic } \\
\text { tissue area }\end{array}$ & Karyotype & $\begin{array}{l}\text { Nucleotide } \\
\text { position }\end{array}$ & $\begin{array}{l}\text { Nucleotide } \\
\text { change }\end{array}$ & $\begin{array}{l}\text { Gene } \\
\text { change }\end{array}$ & $\begin{array}{l}\text { Amino acid } \\
\text { change }\end{array}$ & $\begin{array}{c}\text { Silent } \\
\text { mutation }\end{array}$ & $\begin{array}{c}\text { Frameshift } \\
\text { mutation }\end{array}$ \\
\hline \multirow[t]{2}{*}{1} & $\mathrm{~F} / 29$ & Low & 20 & $46, X X$ & 3834 & $\mathrm{G} \rightarrow \mathrm{A}$ & ND1 & $\mathrm{Ala} \rightarrow \mathrm{Thr}$ & & \\
\hline & & & & & 14178 & $\mathrm{~T} \rightarrow \mathrm{C}$ & ND6 & $\mathrm{Tyr} \rightarrow \mathrm{His}$ & & \\
\hline \multirow[t]{8}{*}{2} & $\mathrm{~F} / 64$ & Extremely low & 20 & $45, \mathrm{XX},-7$ & 1356 & Del A & RNR1 & $\mathrm{SC} \rightarrow \mathrm{SC}$ & + & + \\
\hline & & & & & 2706 & $\mathrm{~A} \rightarrow \mathrm{G}$ & RNR2 & $\mathrm{SC} \rightarrow \operatorname{Trp}$ & & \\
\hline & & & & & 4544 & Del C & ND2 & $\mathrm{Leu} \rightarrow \mathrm{SC}$ & & + \\
\hline & & & & & $4545-4546$ & Ins A & ND2 & $\mathrm{Leu} \rightarrow \mathrm{SC}$ & & + \\
\hline & & & & & 4546 & $\mathrm{G} \rightarrow \mathrm{A}$ & ND2 & $\mathrm{Leu} \rightarrow \mathrm{SC}$ & & \\
\hline & & & & & $12124-12125$ & Ins $\mathrm{C}$ & ND4 & Gly $\rightarrow$ Arg & & + \\
\hline & & & & & 13858 & $\mathrm{~A} \rightarrow \mathrm{T}$ & ND5 & $\mathrm{Thr} \rightarrow \mathrm{Ser}$ & & \\
\hline & & & & & $14099-14100$ & Ins $\mathrm{T}$ & ND5 & Pro $\rightarrow$ Ser & & + \\
\hline \multirow{3}{*}{3} & $\mathrm{M} / 38$ & Extremely low & 10 & $46, X Y$ & 4546 & $\mathrm{G} \rightarrow \mathrm{A}$ & ND2 & Leu $\rightarrow$ Leu & + & \\
\hline & & & & & 13928 & $\mathrm{G} \rightarrow \mathrm{C}$ & ND5 & $\mathrm{Ser} \rightarrow \mathrm{Thr}$ & & \\
\hline & & & & & 14766 & $\mathrm{C} \rightarrow \mathrm{T}$ & CYTB & $\mathrm{Leu} \rightarrow \mathrm{Leu}$ & + & \\
\hline \multirow[t]{2}{*}{4} & $\mathrm{~F} / 24$ & Extremely low & 20 & $46, X X$ & 4164 & $\mathrm{~A} \rightarrow \mathrm{G}$ & ND1 & $\mathrm{Thr} \rightarrow \mathrm{Ala}$ & & \\
\hline & & & & & 10055 & $\mathrm{~A} \rightarrow \mathrm{G}$ & TRNG & Glu $\rightarrow$ Gly & & \\
\hline \multirow[t]{3}{*}{5} & $\mathrm{M} / 50$ & Extremely low & 10 & $46, X Y$ & 4541 & $\mathrm{G} \rightarrow \mathrm{C}$ & ND2 & Ala $\rightarrow$ Pro & & \\
\hline & & & & & 4543 & $\mathrm{~A} \rightarrow \mathrm{T}$ & $\mathrm{ND} 2$ & $\mathrm{Ala} \rightarrow \mathrm{Ala}$ & + & \\
\hline & & & & & 4544 & $\mathrm{C} \rightarrow \mathrm{A}$ & ND2 & $\mathrm{Leu} \rightarrow$ Met & & \\
\hline \multirow[t]{4}{*}{6} & $\mathrm{~F} / 11$ & Low & 20 & $46, X X$ & 4248 & $\mathrm{~T} \rightarrow \mathrm{C}$ & ND1 & $\mathrm{Ser} \rightarrow$ Pro & & \\
\hline & & & & & 4544 & Del C & ND2 & $\mathrm{Leu} \rightarrow \mathrm{SC}$ & & + \\
\hline & & & & & $4545-4546$ & Ins A & ND2 & $\mathrm{Leu} \rightarrow \mathrm{SC}$ & & + \\
\hline & & & & & 4546 & $\mathrm{G} \rightarrow \mathrm{A}$ & ND2 & $\mathrm{Leu} \rightarrow \mathrm{SC}$ & & + \\
\hline 7 & $\mathrm{M} / 29$ & Extremely low & 10 & $46, X Y$ & $15676-15677$ & Ins $\mathrm{C}$ & СYTB & Lys $\rightarrow$ Gln & & + \\
\hline \multirow[t]{9}{*}{8} & $\mathrm{~F} / 52$ & Extremely low & 10 & $46, X X$ & 648 & $\mathrm{~A} \rightarrow \mathrm{C}$ & RNR1 & $\mathrm{Gln} \rightarrow \mathrm{His}$ & & \\
\hline & & & & & 4539 & $\mathrm{~T} \rightarrow \mathrm{C}$ & ND2 & $\mathrm{Leu} \rightarrow$ Pro & & \\
\hline & & & & & 4541 & $\mathrm{G} \rightarrow \mathrm{C}$ & ND2 & $\mathrm{Ala} \rightarrow$ Pro & & \\
\hline & & & & & $12035-12036$ & Ins A & ND4 & Met $\rightarrow$ Asn & & + \\
\hline & & & & & 12037 & $\mathrm{~A} \rightarrow \mathrm{C}$ & ND4 & $\mathrm{SC} \rightarrow \mathrm{Ser}$ & & \\
\hline & & & & & 12040 & Del A & ND4 & $\mathrm{Asn} \rightarrow \mathrm{Thr}$ & & \\
\hline & & & & & 14526 & $\mathrm{~A} \rightarrow \mathrm{C}$ & ND6 & Met $\rightarrow$ Ile & & \\
\hline & & & & & 14572 & $\mathrm{C} \rightarrow \mathrm{T}$ & ND6 & Asn $\rightarrow$ Tyr & & \\
\hline & & & & & $15710-15711$ & Ins $\mathrm{C}$ & CYTB & Aln $\rightarrow$ Pro & & + \\
\hline \multirow[t]{4}{*}{9} & $\mathrm{M} / 45$ & Extremely low & 10 & $46, X Y$ & 4025 & $\mathrm{C} \rightarrow \mathrm{T}$ & ND1 & $\mathrm{Thr} \rightarrow \mathrm{Lle}$ & & \\
\hline & & & & & 4769 & $\mathrm{~A} \rightarrow \mathrm{G}$ & ND2 & Ser $\rightarrow$ Gly & & \\
\hline & & & & & 13860 & $\mathrm{C} \rightarrow \mathrm{T}$ & ND5 & $\mathrm{Thr} \rightarrow \mathrm{Thr}$ & + & \\
\hline & & & & & 14693 & $\mathrm{~A} \rightarrow \mathrm{G}$ & TRNE & $\mathrm{Gln} \rightarrow \mathrm{Gln}$ & + & \\
\hline \multirow[t]{3}{*}{10} & $\mathrm{M} / 37$ & Low & 20 & $46, X Y$ & 6392 & $\mathrm{~T} \rightarrow \mathrm{C}$ & COX1 & Met $\rightarrow$ Lle & & \\
\hline & & & & & 10860 & $\mathrm{~T} \rightarrow \mathrm{G}$ & ND4 & $\mathrm{Ile} \rightarrow \mathrm{Ser}$ & & \\
\hline & & & & & 13859 & $\mathrm{C} \rightarrow \mathrm{T}$ & ND5 & $\mathrm{Thr} \rightarrow$ Lle & & \\
\hline \multirow[t]{2}{*}{11} & $\mathrm{M} / 58$ & Extremely low & 20 & $46, X Y$ & 10861 & $\mathrm{~T} \rightarrow \mathrm{G}$ & ND4 & $\mathrm{Ile} \rightarrow \mathrm{Met}$ & & \\
\hline & & & & & 14370 & Del A & ND6 & $\mathrm{Ile} \rightarrow \mathrm{Ser}$ & & + \\
\hline \multirow[t]{4}{*}{12} & $\mathrm{~F} / 39$ & Extremely low & 20 & $46, X X$ & 4543 & $\mathrm{~A} \rightarrow \mathrm{T}$ & ND2 & $\mathrm{Ala} \rightarrow \mathrm{Ala}$ & + & \\
\hline & & & & & 4544 & $\mathrm{C} \rightarrow \mathrm{T}$ & ND2 & $\mathrm{Leu} \rightarrow \mathrm{Met}$ & & \\
\hline & & & & & 4546 & $\mathrm{G} \rightarrow \mathrm{A}$ & ND2 & $\mathrm{Leu} \rightarrow$ Leu & + & \\
\hline & & & & & 14416 & $\mathrm{~A} \rightarrow \mathrm{G}$ & ND6 & $\mathrm{Gln} \rightarrow \mathrm{Arg}$ & & \\
\hline \multirow[t]{2}{*}{13} & $\mathrm{M} / 29$ & Extremely low & 10 & $46, X Y$ & $14373-14374$ & Ins $\mathrm{C}$ & ND6 & $\mathrm{Leu} \rightarrow$ Leu & + & + \\
\hline & & & & & 14766 & $\mathrm{C} \rightarrow \mathrm{T}$ & CYTB & $\mathrm{Leu} \rightarrow$ Leu & + & \\
\hline \multirow[t]{7}{*}{14} & $\mathrm{~F} / 56$ & Extremely low & 10 & $46, X X$ & 4724 & $\mathrm{C} \rightarrow \mathrm{A}$ & ND2 & $\mathrm{Thr} \rightarrow \mathrm{Thr}$ & + & \\
\hline & & & & & 12020 & $\mathrm{C} \rightarrow \mathrm{G}$ & ND4 & Pro $\rightarrow$ Pro & + & \\
\hline & & & & & 12027 & $\mathrm{~T} \rightarrow \mathrm{C}$ & ND4 & $\mathrm{Leu} \rightarrow$ Leu & + & \\
\hline & & & & & 12028 & $\mathrm{~T} \rightarrow \mathrm{C}$ & ND4 & $\mathrm{Leu} \rightarrow \mathrm{Ser}$ & & \\
\hline & & & & & 12029 & $\mathrm{~A} \rightarrow \mathrm{G}$ & ND4 & Leu $\rightarrow$ Leu & + & \\
\hline & & & & & 12030 & $\mathrm{~A} \rightarrow \mathrm{C}$ & ND4 & $\mathrm{Thr} \rightarrow$ Pro & & \\
\hline & & & & & 12705 & $\mathrm{C} \rightarrow \mathrm{T}$ & ND5 & $\mathrm{Lle} \rightarrow$ Lle & + & \\
\hline 15 & $\mathrm{~F} / 19$ & Extremely low & 10 & $46, X X$ & 4048 & $\mathrm{G} \rightarrow \mathrm{A}$ & ND1 & $\mathrm{Trp} \rightarrow \mathrm{SC}$ & & \\
\hline & & & & & 4532 & $\mathrm{C} \rightarrow \mathrm{T}$ & $\mathrm{ND} 2$ & $\mathrm{Ala} \rightarrow \mathrm{Val}$ & & \\
\hline & & & & & 4539 & $\mathrm{~T} \rightarrow \mathrm{C}$ & $\mathrm{ND} 2$ & $\mathrm{Leu} \rightarrow$ Pro & & \\
\hline & & & & & 4546 & $\mathrm{C} \rightarrow \mathrm{A}$ & ND2 & Leu $\rightarrow$ Leu & + & \\
\hline & & & & & 4883 & $\mathrm{C} \rightarrow \mathrm{T}$ & ND2 & $\mathrm{His} \rightarrow \mathrm{Tyr}$ & & \\
\hline & & & & & 11719 & $\mathrm{G} \rightarrow \mathrm{A}$ & ND4 & $\mathrm{Ala} \rightarrow \mathrm{Thr}$ & & \\
\hline & & & & & 12038 & $\mathrm{~A} \rightarrow \mathrm{T}$ & ND4 & $\mathrm{SC} \rightarrow \mathrm{Tyr}$ & & \\
\hline
\end{tabular}

Continued on next page 
Table 1. Continued.

$\mathrm{F}=$ female; $\mathrm{M}=$ male; $\mathrm{A}=$ adenine; $\mathrm{G}=$ guanine; $\mathrm{C}=$ cytosine; $\mathrm{T}=$ thymine; Ins = insertion; Del = deletion; $N D 1-2$ and 4-6 = NADH dehydrogenase; $R N R 1$ and $2=$ ribosomal RNA; $C Y T B=$ cytochrome $\mathrm{b} ; T R N G=$ tRNA glycine; $T R N E=$ tRNA glutamic acid; $C O X 1=$ cytochrome $\mathrm{c}$ oxidase; $\mathrm{Ala}=$ alanine; $\mathrm{Arg}=$ arginine; $\mathrm{Asn}=$ asparagine; $\mathrm{Gln}=$ glutamine; $\mathrm{Glu}=$ glutamic acid; Gly = glycine; His = histidine; Leu = leucine; Ile = isoleucine; Lys = lysine; Met = methionine; Pro $=$ proline; $\mathrm{Ser}=$ serine; $\mathrm{Thr}=$ threonine; $\mathrm{Tr} \mathrm{p}=$ tryptophan $\mathrm{Tyr}=$ tyrosine; $\mathrm{Val}=$ valine $\mathrm{SC}=$ stop codon.

Table 2. PCR amplification of mtDNA genes.

\begin{tabular}{llcc}
\hline Fragment number & Sequencing primers $\left(5^{\prime} \rightarrow 3^{\prime}\right)$ & Amplicon mtDNA region $(\mathrm{bp})$ & Size $(\mathrm{bp})$ \\
\hline 1 & ACCAAACCCCAAAGACACC (O) & $569-2941$ & 2412 \\
2 & GACTCTAGAATAGGATTGCG (I) & $2797-5193$ & 2436 \\
3 & GTCCTAAACTACCAAACCTG (O) & $5061-7497$ & 2476 \\
& GTGTTAGTCATGTTAGCTTG (I) & $7336-9819$ & 2524 \\
4 & AGCAGTTCTACCGTACAACC (O) & & 2539 \\
5 & TTTGAAAAAGTCATGGAGGC (I) & $9611-12111$ & 2851 \\
6 & GATTTGAGAAGCCTTCGCTT (O) & $11727-14559$ & 1782 \\
7 & GCCAATAATGACGTGAAGTC (I) & $14130-15912$ & 1643 \\
8 & TCCCACTCCTAAACACATC (O) & $15591-16569,1-626$ & \\
\hline
\end{tabular}

$\mathrm{O}=$ outer primer; $\mathrm{I}=$ inner primer.

11 genes, including ND1 (5/8.20\%), ND2 (21/34.43\%), ND4 (13/21.31\%), ND5 (6/9.84\%), ND6 (6/9.84\%), TRNE (1/1.64\%), RNR1 (2/3.28\%), RNR2 (1/1.64\%), COX1 (1/1.64\%), CYTB (4/6.56\%), and TRNG (1/1.64\%). We also found that there were 16 silent mutations $(16 / 26.23 \%)$ and 13 frameshift mutations (13/21.31\%) among all mutations. This information is given in Table 1, Table 3, and Figure 1.

\begin{tabular}{|c|c|c|c|c|c|c|c|c|c|c|c|c|c|c|c|c|}
\hline mtDNA location & 1 & 2 & 3 & 4 & 5 & 6 & 7 & 8 & 9 & 10 & 11 & 12 & 13 & 14 & 15 & Percent \\
\hline RNRI & 0 & 1 & 0 & 0 & 0 & 0 & 0 & 1 & 0 & 0 & 0 & 0 & 0 & 0 & 0 & 3.28 \\
\hline$R N R 2$ & 0 & 1 & 0 & 0 & 0 & 0 & 0 & 0 & 0 & 0 & 0 & 0 & 0 & 0 & 0 & 1.64 \\
\hline$N D 1$ & 1 & 0 & 0 & 1 & 0 & 1 & 0 & 0 & 1 & 0 & 0 & 0 & 0 & 0 & 1 & 8.20 \\
\hline ND2 & 0 & 3 & 1 & 1 & 3 & 3 & 0 & 2 & 1 & 0 & 0 & 3 & 0 & 1 & 3 & 34.43 \\
\hline$C O X 1$ & 0 & 0 & 0 & 0 & 0 & 0 & 0 & 0 & 0 & 1 & 0 & 0 & 0 & 0 & 0 & 1.64 \\
\hline$T R N G$ & 0 & 0 & 0 & 1 & 0 & 0 & 0 & 0 & 0 & 0 & 0 & 0 & 0 & 0 & 0 & 1.64 \\
\hline ND4 & 1 & 1 & 0 & 0 & 0 & 0 & 1 & 3 & 0 & 1 & 1 & 0 & 0 & 5 & 0 & 21.31 \\
\hline ND5 & 0 & 2 & 1 & 0 & 0 & 0 & 0 & 0 & 0 & 1 & 1 & 0 & 0 & 1 & 0 & 9.84 \\
\hline ND6 & 1 & 0 & 0 & 0 & 0 & 0 & 0 & 2 & 0 & 0 & 1 & 1 & 1 & 0 & 0 & 9.84 \\
\hline$T R N E$ & 0 & 0 & 0 & 0 & 0 & 0 & 0 & 0 & 1 & 0 & 0 & 0 & 0 & 0 & 0 & 1.64 \\
\hline CYTB & 0 & 0 & 1 & 0 & 0 & 0 & 1 & 1 & 0 & 0 & 0 & 0 & 1 & 0 & 0 & 6.56 \\
\hline
\end{tabular}

For abbreviations, see legend to Table 1.

Mitochondrial sequencing indicated that these mutations were found in the coding region closely related to the mitochondrial oxidative respiratory chain, covering ND1-2 and 4-6, CYTB, and other related genes, and that mutation levels were particularly high in ND2 and 


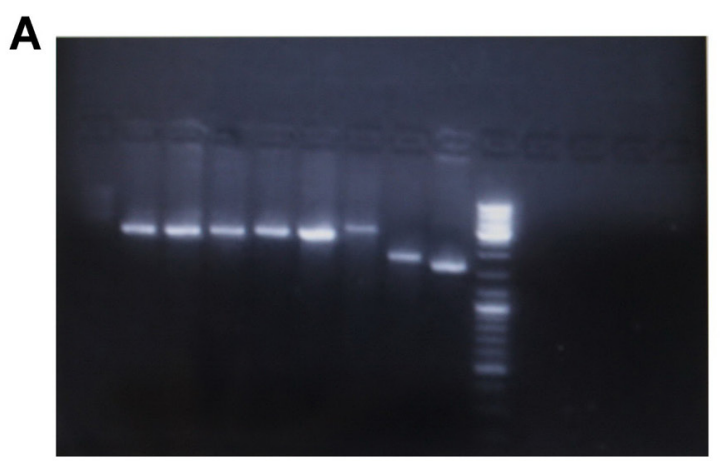

C

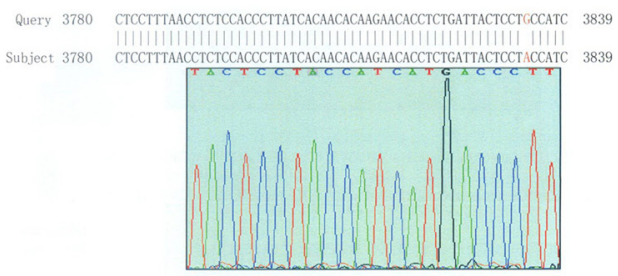

$\mathbf{E}$

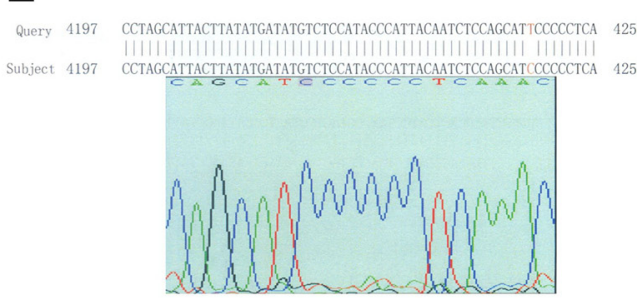

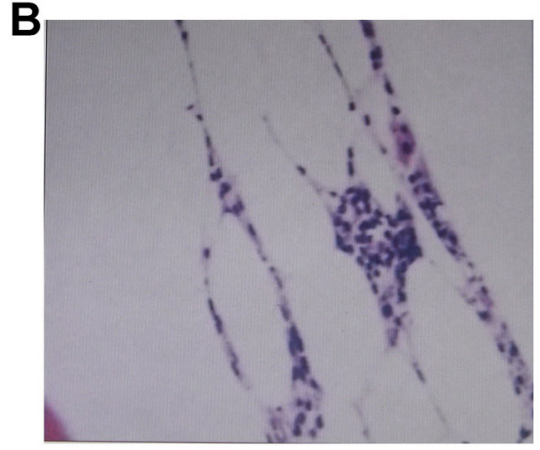

D

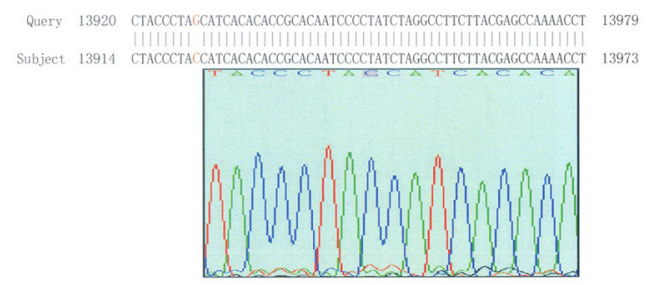

$\mathbf{F}$

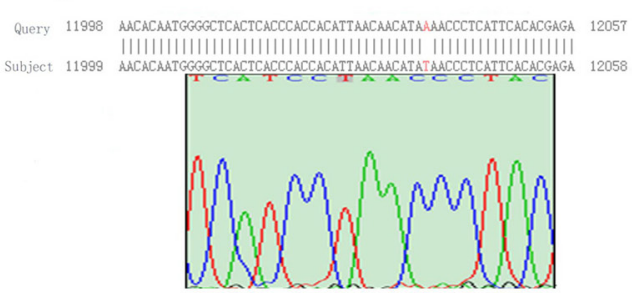

Figure 1. Electropherogram, bone marrow biopsy picture, and mutation of mtDNA. A. Electropherogram of 8 fragments; B. bone marrow biopsy of patient 1; C. G3834A mutation (arrow) was found in patient 1; D. G13928C mutation was found in patient 3; E. T4248C mutation was found in patient 6; F. A12038T mutation was found in patient 15; Query = revised Cambridge Reference Sequence; Subject = sequence of patients.

ND4. We speculate that this may be an important reason behind the failure of hematopoietic stem cells.

\section{mtDNA polymorphisms in AA patients}

In this study, we detected 162 polymorphisms in 15 genomes, including the D-loop region (88/54.32\%), RNR1 (18/11.11\%), RNR2 (8/4.94\%), ND1 (2/1.23\%), ND2 (7/4.32\%), COX1 (8/4.94\%), COX2 (1/0.62\%), COX3 (1/0.62\%), ATP6 (2/1.23\%), ND3 (2/1.23\%), ND4L (2/1.23\%), ND4 (4/2.47\%), ND5 (8/4.94\%), ND6 (1/0.62\%), and CYTB (10/6.17\%) (Table 4). Interestingly, the majority of these polymorphisms were in the D-loop region, where numerous mutations involved in leukemia and myelodysplastic syndromes are found. However, in this study, no new polymorphisms were found. 
Table 4. mtDNA polymorphisms detected in patients with aplastic anemia.

\begin{tabular}{lcccccccccccccccc}
\hline mtDNA location & 1 & 2 & 3 & 4 & 5 & 6 & 7 & 8 & 9 & 10 & 11 & 12 & 13 & 14 & 15 & Percent \\
\hline D-loop & 6 & 8 & 9 & 9 & 13 & 13 & 4 & 8 & 3 & 2 & 1 & 0 & 5 & 5 & 2 & 54.32 \\
RNR1 & 1 & 3 & 2 & 1 & 4 & 0 & 2 & 0 & 0 & 4 & 0 & 0 & 1 & 0 & 0 & 11.11 \\
RNR2 & 0 & 1 & 0 & 0 & 3 & 0 & 1 & 0 & 0 & 2 & 0 & 0 & 0 & 1 & 0 & 4.94 \\
ND1 & 0 & 1 & 0 & 0 & 0 & 0 & 0 & 0 & 0 & 1 & 0 & 0 & 0 & 0 & 0 & 1.23 \\
ND2 & 0 & 1 & 0 & 0 & 0 & 3 & 1 & 0 & 0 & 0 & 0 & 0 & 0 & 2 & 0 & 4.32 \\
COX1 & 0 & 1 & 1 & 2 & 0 & 0 & 0 & 0 & 0 & 0 & 2 & 0 & 0 & 0 & 2 & 4.94 \\
COX2 & 0 & 1 & 0 & 0 & 0 & 0 & 0 & 0 & 0 & 0 & 0 & 0 & 0 & 0 & 0 & 0.62 \\
COX3 & 0 & 0 & 0 & 0 & 0 & 0 & 0 & 0 & 0 & 0 & 1 & 0 & 0 & 0 & 0 & 0.62 \\
ATP6 & 0 & 0 & 0 & 0 & 0 & 1 & 0 & 0 & 1 & 0 & 0 & 0 & 0 & 0 & 0 & 1.23 \\
ND3 & 0 & 0 & 0 & 0 & 0 & 1 & 0 & 0 & 1 & 0 & 0 & 0 & 0 & 0 & 0 & 1.23 \\
ND4L & 0 & 0 & 0 & 0 & 0 & 0 & 0 & 0 & 2 & 0 & 0 & 0 & 0 & 0 & 0 & 1.23 \\
ND4 & 0 & 2 & 0 & 0 & 0 & 2 & 0 & 0 & 0 & 0 & 0 & 0 & 0 & 0 & 0 & 2.47 \\
ND5 & 1 & 4 & 0 & 0 & 0 & 0 & 0 & 0 & 0 & 0 & 1 & 2 & 0 & 0 & 0 & 4.94 \\
ND6 & 0 & 1 & 0 & 0 & 0 & 0 & 0 & 0 & 0 & 0 & 0 & 0 & 0 & 0 & 0 & 0.62 \\
CYTB & 1 & 0 & 0 & 2 & 0 & 1 & 0 & 1 & 1 & 0 & 1 & 2 & 0 & 0 & 1
\end{tabular}

ATP6 $=$ adenosine triphosphate 6; ND4L = NADH dehydrogenase, subunit 4L. For other abbreviations, see legend to Table 1.

\section{DISCUSSION}

Acquired mtDNA deletions in the hematopoietic compartment have also been observed to occur in association with severe pancytopenia and reticulocytopenia (Clayton, 1984). In this study, we detected 61 mutations in 11 genes, including ND1 (5/8.20\%), ND2 (21/34.43\%), ND4 (13/21.31\%), ND5 (6/9.84\%), ND6 (6/9.84\%), TRNE (1/1.64\%), RNR1 (2/3.28\%), RNR2 (1/1.64\%), COX1 (1/1.64\%), CYTB (4/6.56\%), and TRNG (1/1.64\%). Also, the mutation rate was particularly high in ND2 (15/33.33\%) and ND4 (10/22.22\%) in patients' mtDNA genome, excepting these silent mutations. These genes are closely linked to oxidation in the respiratory chain. Interestingly, there were no D-loop mutations in these heteroplasmic mutations.

ND1-2 and 4-6, and CYTB are important components of NADH-ubiquinone oxidoreductase (Complex I) and ubiquinone cytochrome c oxidoreductase (Complex III). Mitochondrial injury is reflected by mtDNA damage as well as by a decline in the levels of mtRNA transcripts, protein synthesis, and mitochondrial function. A decrease in these complex activities can result in a decrease in cellular energy, disruption of cell signaling, and interference with cellular differentiation and apoptosis. Furthermore, deficient mitochondrial ATP production due to mtDNA mutation may promote chromosomal instability (Gattermann, 2004). Cells with an inadequate ATP supply may have difficulty in correctly segregating their chromosomes during mitosis. These factors may result in a decrease in energy metabolism, which will affect self-renewal and differentiation of the hematopoietic stem cell.

Interestingly, the genes affected were involved in oxidative phosphorylation. In contrast to the mutations involved in hematologic malignancies such as acute myeloblastic leukemia and myelodysplastic syndromes, these mutations were mainly in the D-loop. In these studies, a great deal of nucleotide alterations were detected in the D-loop region in acute myeloblastic leukemia and myelodysplastic syndrome patients, suggesting that the D-loop is a mutational hotspot in human cancer. It was found that leukemia cells utilize glycolysis more vigorously than oxidative phosphorylation in mitochondria (Gattermann, 2000; Shin et al., 2003; Grist et al., 2004; Suganuma et al., 2010). This may be caused by differences between benign and malignant hematologic disorders. 
In summary, the mutations occurred in the region that influences the replication and transcription of mtDNA and thus are likely to have impact on function, increasing the permeability of the mitochondrial inner membrane and destroying the membrane potential, which would then trigger caspase activity and eventually cause cell apoptosis. Therefore, the functional defect caused by mtDNA mutations may be the primary cause of bone marrow failure in AA.

\section{ACKNOWLEDGMENTS}

Research supported by the Shandong Province Technology Development Program of Traditional Chinese Medicine (\#2011-063), the Natural Science Foundation of Shandong Province (\#ZR2012HQ023), the Chinese National Key Technologies R\&D Program during the 11th Five-Year Plan Period (\#2007BAI20B06), and the Affiliated Hospital of Shandong University of Traditional Chinese Medicine. We thank Sangon Biotech (Shanghai) Co., Ltd. for their work on the mtDNA sequence.

\section{Conflict of interests}

The authors have declared that no conflict of interest exists.

\section{REFERENCES}

Andrews RM, Kubacka I, Chinnery PF, Lightowlers RN, et al. (1999). Reanalysis and revision of the Cambridge reference sequence for human mitochondrial DNA. Nat. Genet. 23: 147.

Bohr VA, Stevnsner T and de Souza-Pinto NC (2002). Mitochondrial DNA repair of oxidative damage in mammalian cells. Gene 286: 127-134.

Chen D, Cao G, Hastings T, Feng Y, et al. (2002). Age-dependent decline of DNA repair activity for oxidative lesions in rat brain mitochondria. $J$. Neurochem. 81: 1273-1284.

Chinnery PF and Schon EA (2003). Mitochondria. J. Neurol. Neurosurg. Psychiatr. 74: 1188-1199.

Clayton DA (1984). Transcription of the mammalian mitochondrial genome. Annu. Rev. Biochem. 53: 573-594.

DiMauro S and Schon EA (2003). Mitochondrial respiratory-chain diseases. N. Engl. J. Med. 348: 2656-2668.

Gattermann N (2000). From sideroblastic anemia to the role of mitochondrial DNA mutations in myelodysplastic syndromes. Leuk. Res. 24: 141-151.

Gattermann N (2004). Mitochondrial DNA mutations in the hematopoietic system. Leukemia 18: 18-22.

Grist SA, Lu XJ and Morley AA (2004). Mitochondrial mutations in acute leukaemia. Leukemia 18: 1313-1316.

Hatfill SJ, La Cock CJ, Laubscher R, Downing TG, et al. (1993). A role for mitochondrial DNA in the pathogenesis of radiation-induced myelodysplasia and secondary leukemia. Leuk. Res. 17: 907-913.

Lee MS and Levin BC (2002). MitoAnalyzer, a computer program and interactive web site to determine the effects of single nucleotide polymorphisms and mutations in human mitochondrial DNA. Mitochondrion 1: 321-326.

Linnartz B, Anglmayer R and Zanssen S (2004). Comprehensive scanning of somatic mitochondrial DNA alterations in acute leukemia developing from myelodysplastic syndromes. Cancer Res. 64: 1966-1971.

Penta JS, Johnson FM, Wachsman JT and Copeland WC (2001). Mitochondrial DNA in human malignancy. Mutat. Res. 488: 119-133.

Richter C, Park JW and Ames BN (1988). Normal oxidative damage to mitochondrial and nuclear DNA is extensive. Proc. Natl. Acad. Sci. U. S. A. 85: 6465-6467.

Shadel GS and Clayton DA (1997). Mitochondrial DNA maintenance in vertebrates. Annu. Rev. Biochem. 66: 409-435.

Shin MG, Kajigaya S, Levin BC and Young NS (2003). Mitochondrial DNA mutations in patients with myelodysplastic syndromes. Blood 101: 3118-3125.

Suganuma K, Miwa H, Imai N, Shikami M, et al. (2010). Energy metabolism of leukemia cells: glycolysis versus oxidative phosphorylation. Leuk. Lymphoma 51: 2112-2119.

Wulfert M, Kupper AC, Tapprich C, Bottomley SS, et al. (2008). Analysis of mitochondrial DNA in 104 patients with myelodysplastic syndromes. Exp. Hematol. 36: 577-586. 